\title{
SYMMETRIC PERIODIC ORBITS NEAR HETEROCLINIC LOOPS AT INFINITY FOR A CLASS OF POLYNOMIAL VECTOR FIELDS
}

\author{
MONTSERRAT CORBERA \\ Departament d'Informàtica i Matemàtiques, Universitat de Vic, \\ 08500 Vic, Barcelona, Spain. E-mail: montserrat.corbera@uvic.es \\ JAUME LLIBRE \\ Departament de Matemàtiques, Universitat Autònoma de Barcelona, \\ 08193 Bellaterra, Barcelona, Spain.E-mail: jllibre@mat.uab.es
}

\begin{abstract}
For polynomial vector fields in $\mathbb{R}^{3}$, in general, it is very difficult to detect the existence of an open set of periodic orbits in their phase portraits. Here, we characterize a class of polynomial vector fields of arbitrary even degree having an open set of periodic orbits. The main two tools for proving this result are, first, the existence in the phase portrait of a symmetry with respect to a plane and, second, the existence of two symmetric heteroclinic loops.
\end{abstract}




\section{Introduction and main result}

In this paper we will study the periodic orbits near infinity of a class of polynomial vector fields in $\mathbb{R}^{3}$. In order to study the behaviour of a polynomial vector field near infinity we will use the Poincaré compactification (see Section 2 for details). This technique allows us to extend the vector field in $\mathbb{R}^{3}$ to a unique analytic vector field on the Poincaré sphere $\mathbb{S}^{3}$ and on the Poincaré ball $\mathbb{D}^{3}$, whose boundary, the sphere $\mathbb{S}^{2}$, plays the role of the infinity for the initial polynomial vector field.

Let $P, Q$ and $R$ be polynomials in the variables $x, y$ and $z$. We consider the polynomial vector field $X=(P, Q, R)$ in $\mathbb{R}^{3}$ that satisfies the following conditions

$\left(C_{1}\right)$ The flow of $X$ is invariant under the symmetry $(x, y, z, t) \longrightarrow(-x, y, z,-t)$. So the phase portrait of $X$ is symmetric with respect to the plane $x=0$.

$\left(C_{2}\right)$ The maximum of the degrees of $P, Q$ and $R$ is called the degree of $X$. Here we assume that this degree is even and equal to $n$.

$\left(C_{3}\right)$ The straight line $y=z=0$ is invariant by the flow of $X$, it does not contain any singular point and the flow on it goes in the increasing direction of the $x$-axis.

$\left(C_{4}\right)$ The straight line $y=z=0$ intersects the boundary of the Poincaré ball at two singular points $a$ and $b$, the origins of the local charts $U_{1}$ and $V_{1}$ respectively. Moreover, $a$ is hyperbolic with local unstable manifold $z_{2}=z_{3}=0$ in the local chart $U_{1}$. Recall that a singular point is hyperbolic if the real part of all its eigenvalues is different from zero. Note that by the symmetry of the problem the singular point $b$ is also hyperbolic.

$\left(C_{5}\right)$ The straight line $z_{2}=z_{3}=0$ of the local chart $U_{2}$ of the Poincaré ball is invariant by the flow of $X$, it does not contain any singular point and the flow on it goes in the decreasing direction of the $z_{1}$-axis.

We note that conditions $\left(C_{2}\right)-\left(C_{5}\right)$ say that the Poincaré compactification of $X, p(X)$, possesses a heteroclinic loop $\mathcal{L}$ which is formed by two singular points at infinity (the points $a$ and $b$ ) connected by

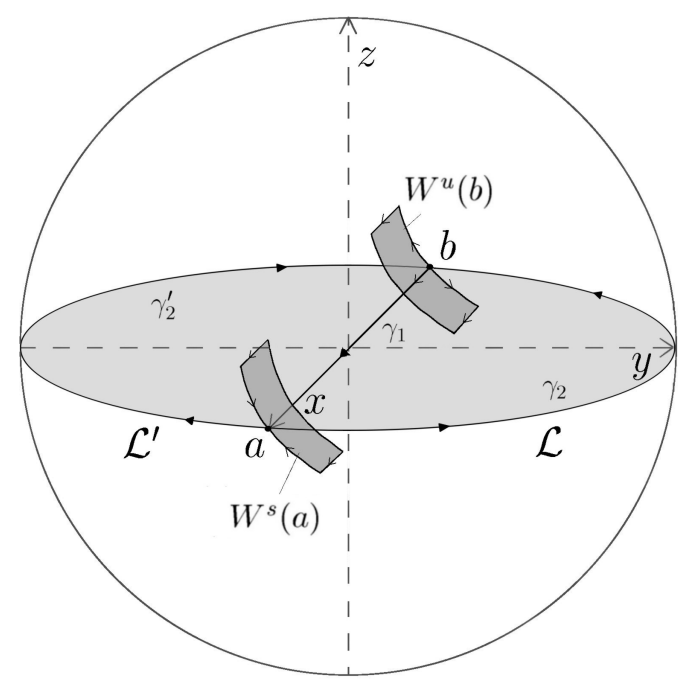

Fig. 1. The heteroclinic loops $\mathcal{L}$ and $\mathcal{L}^{\prime}$ and the Poincaré ball $\mathbb{D}^{3}$.

the straight line $y=z=0$ in $\mathbb{R}^{3}$ and the straight line $z_{2}=z_{3}=0$ at infinity in the local chart $U_{2}$, see Fig. 1 .

Recall that the flow on the infinity $\mathbb{S}^{2}$ is symmetric with respect to the origin of $\mathbb{S}^{2}$. This symmetry reverses the orientation of the orbits because the degree $n$ is even. Due to this symmetry on $\mathbb{S}^{2}$, $p(X)$ possesses another heteroclinic loop $\mathcal{L}^{\prime}$ which is formed by the two previous singular points at infinity connected by the straight line $y=z=0$ in $\mathbb{R}^{3}$ and the straight line $z_{2}=z_{3}=0$ at infinity in the local chart $V_{2}$.

On the other hand, as we will see in Section 3, if an orbit $\varphi$ crosses the plane $x=0$ in two different points, then using the symmetry $\left(C_{1}\right) \varphi$ is a symmetric periodic orbit.

Using the symmetry $\left(C_{1}\right)$ and the heteroclinic loops $\mathcal{L}$ and $\mathcal{L}^{\prime}$ we get our main result.

Theorem 1.1. Let $X$ be a polynomial vector field in $\mathbb{R}^{3}$ satisfying conditions $\left(C_{1}\right)-\left(C_{5}\right)$. Let $D_{\varepsilon}$ denote the punctured disc $\left\{0<y_{0}^{2}+z_{0}^{2}<\varepsilon^{2}\right\}$ minus a differentiable curve $\gamma$ that passes through the origin of the disc and separates it into two components as in Fig. 2.

(a) There exists $\varepsilon>0$ sufficiently small such that the solutions of $X$ having initial conditions $x(0)=0, y(0)=y_{0}$ and $z(0)=z_{0}$, with $\left(y_{0}, z_{0}\right) \in D_{\varepsilon}$ are periodic solutions that lie near one of the heteroclinic loops $\mathcal{L}$ and $\mathcal{L}^{\prime}$. 


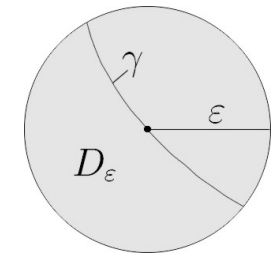

Fig. 2. The punctured disc $D_{\varepsilon}$.

(b) The 2-dimensional unstable manifold of the singular point $b, W^{u}(b)$, coincides with the 2-dimensional stable manifold of the singular point $a, W^{s}(a)$.

The class of polynomial vector fields satisfying conditions $\left(C_{1}\right)-\left(C_{5}\right)$ is not empty. In particular, we shall prove the following result.

Theorem 1.2. A polynomial vector field $X=$ $(P, Q, R)$ in $\mathbb{R}^{3}$ satisfies conditions $\left(C_{1}\right)-\left(C_{5}\right)$ if and only if

$$
\text { (i) } \begin{aligned}
P & =\sum_{\substack{0 \leqslant i+j+k \leqslant n \\
\text { i even }}} a_{i j k} x^{i} y^{j} z^{k}, \\
Q & =\sum_{\substack{0 \leqslant i+j+k \leqslant n \\
i \text { odd, } j+k \geqslant 1}} b_{i j k} x^{i} y^{j} z^{k}, \\
R & =\sum_{\substack{0 \leqslant i+j+k \leqslant n \\
i \text { odd }, j+k \geqslant 1}} c_{i j k} x^{i} y^{j} z^{k} ;
\end{aligned}
$$

(ii) $\sum_{\substack{0 \leqslant i \leqslant n \\ i \text { even }}} a_{i 00} x^{i}>0$ for all $x \in \mathbb{R}$;

(iii) $a_{n 00}>0$;

(iv) $c_{i, n-i, 0}=0$ for all odd $i \in\{1, \ldots, n-1\}$;

(v) $b_{n-1,1,0}>a_{n 00}>c_{n-1,0,1}$;

$$
\begin{aligned}
& \text { (vi) } \sum_{\substack{i+j+k \leqslant n \\
i \text { even }}} a_{i, n-i, 0} z_{1}^{i}-\sum_{\substack{0 \leqslant i+j+k \leqslant n \\
i \text { odd }}} b_{i, n-i, 0} z_{1}^{i+1} \text { is } \\
& \text { negative for all } z_{1} \in \mathbb{R} \text {. }
\end{aligned}
$$

Let $U$ be an open subset of $\mathbb{R}^{3}$. A non-constant function $H: U \longrightarrow \mathbb{R}$ is called a first integral of $X$ if it is constant on every solution of $X$ contained in $U$. Then a function $H \in \mathcal{C}^{1}(U)$ is a first integral of $X$ on $U$ if and only if

$$
\frac{\partial H}{\partial x} P+\frac{\partial H}{\partial y} Q+\frac{\partial H}{\partial z} R=0 .
$$

Two first integrals $H_{1}$ and $H_{2}$ defined in $U$ are independent if for all $(x, y, z) \in U$, except perhaps in a subset of zero Lebesgue measure, their gradients are linearly independent.

A vector field in $\mathbb{R}^{3}$ is called integrable if it has two independent first integrals. For quadratic polynomial vector fields in $\mathbb{R}^{3}$ satisfying conditions $\left(C_{1}\right)-\left(C_{5}\right)$ we get the following result.

Proposition 1.3. Let $D_{\varepsilon}$ be defined as in Theorem 1.1. For the polynomial differential systems

$$
\begin{aligned}
\dot{x}= & a_{000}+a_{200} x^{2}+a_{010} y+a_{020} y^{2}+a_{001} z+ \\
& a_{011} y z+a_{002} z^{2}, \\
\dot{y}= & b_{110} x y+b_{101} x z \\
\dot{z}= & c_{101} x z
\end{aligned}
$$

with $a_{000}>0, a_{020}<0, b_{110}>a_{200}>0$ and $c_{101}<$ $a_{200}$, we can find $\varepsilon>0$ sufficiently small such that their solutions with initial conditions $x(0)=0$, $y(0)=y_{0}$ and $z(0)=z_{0}$, with $\left(y_{0}, z_{0}\right) \in D_{\varepsilon}$, are periodic solutions with very large periods. Moreover all these systems are integrable.

The fact that all quadratic vector fields satisfying conditions $\left(C_{1}\right)-\left(C_{5}\right)$ are integrable (see Proposition 1.3) induces the following natural question.

Open question: Are the polynomial vector fields satisfying conditions $\left(C_{1}\right)-\left(C_{5}\right)$ having arbitrary even degree integrable?

The periodic orbits of polynomial vector fields near different kinds of heteroclinic loops having two singular points at infinity and two straight lines orbits connecting them (finite or not) have been studied by several authors, see for instance [Buzzi et al., 2004], [Llibre et. al, 2004] and [Newell et. al, 1988]. We note that a big difference between our system and these systems previously studied is that we have a symmetry with respect to a plane, and all these other systems have a symmetry with respect to a straight line.

This paper is organized as follows. In Section 2 we describe the Poincaré compactification for polynomial vector fields in $\mathbb{R}^{3}$. In Section 3 we prove Theorem 1.1. In Section 4 we prove Theorem 1.2. Finally in Section 5 we analyze the class of quadratic vector fields satisfying conditions $\left(C_{1}\right)-$ $\left(C_{5}\right)$. In particular, we will prove Proposition 1.3, 
and we also will describe the global phase portrait of a particular example of this class of quadratic vector fields.

\section{The Poincaré compactification in $\mathbb{R}^{3}$}

A polynomial vector field $X$ in $\mathbb{R}^{n}$ can be extended to a unique analytic vector field on the sphere $\mathbb{S}^{n}$. The technique for making such an extension is called the Poincaré compactification. The Poincaré compactification allows us to study the vector field in a neighbourhood of infinity which is represented by the equator $\mathbb{S}^{n-1}$ of the sphere $\mathbb{S}^{n}$. Poincaré introduced this technique for polynomial vector fields in $\mathbb{R}^{2}$, its extension to $\mathbb{R}^{n}$ can be found in [Cima et al., 1990]. Here we only consider the Poincaré compactification for polynomial vector fields in $\mathbb{R}^{3}$.

Let $X=\left(P^{1}, P^{2}, P^{3}\right)$ be a polynomial vector field in $\mathbb{R}^{3}$, let $x=\left(x_{1}, x_{2}, x_{3}\right)$ and let $m=$ $\max \left\{\operatorname{deg}\left(P^{1}\right), \operatorname{deg}\left(P^{2}\right), \operatorname{deg}\left(P^{3}\right)\right\}$ be the degree of $X$.

We consider the unit sphere in $\mathbb{R}^{4}, \mathbb{S}^{3}=\{y=$ $\left.\left(y_{1}, y_{2}, y_{3}, y_{4}\right) \in \mathbb{R}^{4}:\|y\|=1\right\}$, which is called the Poincaré sphere; and we consider the hyperplane $\Pi=\left\{\left(x_{1}, x_{2}, x_{3}, x_{4}\right) \in \mathbb{R}^{4}: x_{4}=1\right\}$ which is the tangent to $\mathbb{S}^{3}$ at the northern pole $(0,0,0,1)$. We note that $\Pi$ is diffeomorphic to $\mathbb{R}^{3}$, then we identify $\mathbb{R}^{3}$ with $\Pi$. Let $H_{+}=\left\{y \in \mathbb{S}^{3}: y_{4}>0\right\}$ and $H_{-}=\left\{y \in \mathbb{S}^{3}: y_{4}<0\right\}$ be the northern and southern hemispheres of $\mathbb{S}^{3}$, respectively.

We consider the central projections $f_{+}: \Pi=$ $\mathbb{R}^{3} \longrightarrow H_{+}$and $f_{-}: \Pi=\mathbb{R}^{3} \longrightarrow H_{-}$, defined by $f_{+}(x)=\left(x_{1}, x_{2}, x_{3}, 1\right) / \Delta(x)$ and $f_{-}(x)=$ $-\left(x_{1}, x_{2}, x_{3}, 1\right) / \Delta(x)$ respectively, where $\Delta(x)=$ $\left(1+\sum_{i=1}^{3} x_{i}^{2}\right)^{1 / 2}$. Through these central projections, $\mathbb{R}^{3}$ can be identified with the northern and southern hemispheres of $\mathbb{S}^{3}$ respectively. So the vector field $X$ induces a vector field $\widetilde{X}$ in $H_{+} \cup H_{-}$ defined by $\widetilde{X}(y)=\left(D f_{+}\right)_{x} X(x)$ when $y=f_{+}(x)$, and by $\widetilde{X}(y)=\left(D f_{-}\right)_{x} X(x)$ when $y=f_{-}(x)$.

We note that $\widetilde{X}(y)$ gives two copies of $X$ one on the northern hemisphere $H_{+}$and the other one on the southern hemisphere $H_{-}$. Moreover $\widetilde{X}(y)$ is defined in $H_{+} \cup H_{-}$, but in general it is not defined on the equator $\mathbb{S}^{2}=\left\{y \in \mathbb{S}^{3}: y_{4}=0\right\}$ of $\mathbb{S}^{3}$. We can extend analytically the vector field $\widetilde{X}(y)$ to the whole sphere $\mathbb{S}^{3}$ in the following way

$$
p(X)(y)=y_{4}^{m-1} \widetilde{X}(y) .
$$

The vector field $p(X)$ is called the Poincaré compatification of $X$.

The closed northern hemisphere is a closed ball of $\mathbb{R}^{3}$, called the Poincaré ball $\mathbb{D}^{3}$, its interior is diffeomorphic to $\mathbb{R}^{3}$ and its boundary $\mathbb{S}^{2}$ correspond to the infinity of $\mathbb{R}^{3}$. We note that the boundary of the Poincaré ball is invariant by the flow of $p(X)$. So $p(X)$ allows us to study the behaviour of $X$ in a neighbourhood of infinity.

To compute the analytical expression for $p(X)$ we shall consider $\mathbb{S}^{3}$ as a differentiable manifold and we choose the eight coordinate neighbourhoods $U_{i}=\left\{y \in S^{3}: y_{i}>0\right\}$ and $V_{i}=\left\{y \in S^{3}: y_{i}<0\right\}$, for $i=1, \ldots, 4$, with the corresponding coordinate maps $F_{i}: U_{i} \longrightarrow \mathbb{R}^{3}$ and $G_{i}: V_{i} \longrightarrow \mathbb{R}^{3}$ defined by

$$
F_{i}(y)=G_{i}(y)=\frac{1}{y_{i}} \bar{y}_{i}=\left(z_{1}, z_{2}, z_{3}\right),
$$

where $\bar{y}_{i}$ is the point $\left(y_{1}, y_{2}, y_{3}, y_{4}\right)$ without the component $y_{i}$.

We do the computations of $p(X)$ on the local chart $U_{1}$. The coordinate map on $U_{1}$ is given by $F_{1}(y)=\left(y_{2} / y_{1}, y_{3} / y_{1}, y_{4} / y_{1}\right)=\left(z_{1}, z_{2}, z_{3}\right)$. We note that the map $F_{1}$ is the inverse of the central projection from the origin to the tangent space of $\mathbb{S}^{3}$ at the point $(1,0,0,0)$. The expression of $p(X)$ in this local chart $U_{1}$ is given by $\left(D F_{1}\right)_{y}(p(X)(y))$, which after doing the computations becomes

$$
\frac{z_{3}^{m}}{(\Delta z)^{m-1}}\left(-z_{1} P^{1}+P^{2},-z_{2} P^{1}+P^{3},-z_{3} P^{1}\right),
$$

where $P^{i}=P^{i}\left(1 / z_{3}, z_{1} / z_{3}, z_{2} / z_{3}\right)$ and $\Delta z=(1+$ $\left.\sum_{i=1}^{3} z_{i}^{2}\right)^{1 / 2}$.

In a similar way we can deduce the expressions for $p(X)$ in the local charts $U_{2}$ and $U_{3}$. These are

$$
\frac{z_{3}^{m}}{(\Delta z)^{m-1}}\left(-z_{1} P^{2}+P^{1},-z_{2} P^{2}+P^{3},-z_{3} P^{2}\right),
$$

where $P^{i}=P^{i}\left(z_{1} / z_{3}, 1 / z_{3}, z_{2} / z_{3}\right)$, and

$$
\frac{z_{3}^{m}}{(\Delta z)^{m-1}}\left(-z_{1} P^{3}+P^{1},-z_{2} P^{3}+P^{2},-z_{3} P^{3}\right),
$$

where $P^{i}=P^{i}\left(z_{1} / z_{3}, z_{2} / z_{3}, 1 / z_{3}\right)$, respectively.

The expression for $p(X)$ in the local chart $U_{4}$ is $(\Delta z)^{1-m}\left(P^{1}, P^{2}, P^{3}\right)$ where $P^{i}=P^{i}\left(z_{1}, z_{2}, z_{3}\right)$. Finally, the expression for $p(X)$ in the local charts $V_{i}$ is the same as in $U_{i}$ multiplied by $(-1)^{m-1}$.

We note that with a convenient change of the time we shall omit the factor $1 /(\Delta z)^{m-1}$ in the expressions of $p(X)$. 


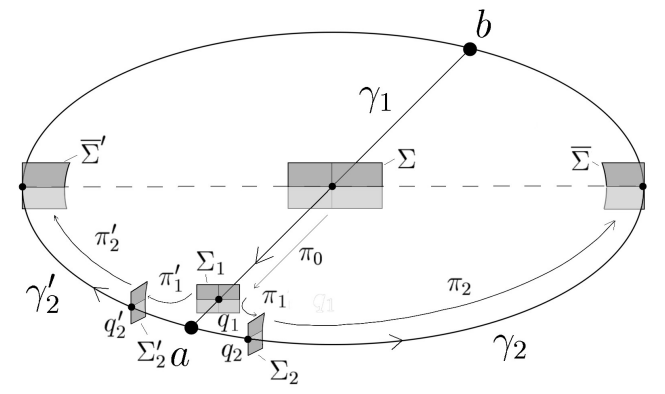

Fig. 3. The Poincaré maps $\pi: \Sigma \longrightarrow \bar{\Sigma}$ and $\pi^{\prime}$ : $\Sigma \longrightarrow \bar{\Sigma}^{\prime}$.

\section{Proof of Theorem 1.1}

Let $X$ be a polynomial vector field in $\mathbb{R}^{3}$ satisfying conditions $\left(C_{1}\right)-\left(C_{5}\right)$. Recall that $a$ and $b$ are the origins of the local charts $U_{1}$ and $V_{1}$ of the Poincaré ball $\mathbb{D}^{3}$, respectively. Let $\gamma_{1}$ be the straight line $y=z=0$ in $\mathbb{R}^{3}$, and let $\gamma_{2}$ (respectively, $\gamma_{2}^{\prime}$ ) be the straight line at infinity $z_{2}=z_{3}=0$ in the local chart $U_{2}$ (respectively, $V_{2}$ ). From conditions $\left(C_{2}\right)$ $\left(C_{5}\right)$, the vector field $p(X)$ possesses two heteroclinic loops $\mathcal{L}$ and $\mathcal{L}^{\prime}$ formed by the singular points $a$ and $b$ connected by the straight lines $\gamma_{1}, \gamma_{2}$ and $\gamma_{1}$, $\gamma_{2}^{\prime}$, respectively, see Fig. 1 . We denote by $\varphi(t, q)$ the flow generated by system $X$, satisfying $\varphi(0, q)=q$.

We consider the cross section $\bar{\Sigma}=$ $\left\{\left(z_{1}, z_{2}, z_{3}\right) \in U_{2} \cap \operatorname{Int}\left(\mathbb{D}^{3}\right): z_{1}=0\right\}$ to the orbit $\gamma_{2}$ at the origin of the local chart $U_{2}$, and the cross section $\bar{\Sigma}^{\prime}=\left\{\left(z_{1}, z_{2}, z_{3}\right) \in V_{2} \cap \operatorname{Int}\left(\mathbb{D}^{3}\right): z_{1}=0\right\}$ to the orbit $\gamma_{2}^{\prime}$ at the origin of the local chart $V_{2}$, see Fig. 3. As usual $\operatorname{Int}\left(\mathbb{D}^{3}\right)$ denotes the interior of the Poincaré ball $\mathbb{D}^{3}$. In a neighbourhood of the origin $a$ of the local chart $U_{1}$ we take three cross sections: a cross section $\Sigma_{1}$ to the orbit $\gamma_{1}$ at a point $q_{1} \in \gamma_{1}$ near $a$, a cross section $\Sigma_{2}$ to the orbit $\gamma_{2}$ at a point $q_{2} \in \gamma_{2}$ near $a$, and a cross section $\Sigma_{2}^{\prime}$ to the orbit $\gamma_{2}^{\prime}$ at a point $q_{2}^{\prime} \in \gamma_{2}^{\prime}$ near $a$. Finally, we consider the cross section $\Sigma=\left\{(x, y, z) \in \mathbb{R}^{3}: x=0\right\}$ to the orbit $\gamma_{1}$ at the origin.

We define two Poincaré maps $\pi: \Sigma \longrightarrow \bar{\Sigma}$ and $\pi^{\prime}: \Sigma \longrightarrow \bar{\Sigma}^{\prime}$ in the following way. We consider the diffeomorphism $\pi_{0}: \Sigma \rightarrow \Sigma_{1}$ defined by $\pi_{0}(q)=p$, where $p$ is the point at which the orbit $\varphi(t, q)$ intersects the cross section $\Sigma_{1}$ for the first time. By the continuity of the flow $\varphi$ with respect to initial conditions, if $q$ is sufficiently close to the origin of $\Sigma$, then the orbit $\varphi(t, q)$ is close to the orbit $\gamma_{1}$ for

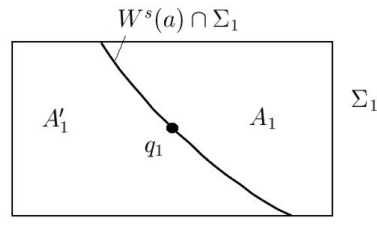

Fig. 4. The sets $A_{1}$ and $A_{1}^{\prime}$ restricted to a rectangle sufficiently small contained in $\Sigma_{1}$.

all $t$ in a finite interval of time. Since the orbit $\gamma_{1}$ expends a finite time for going from the origin to $q_{1}$, we can guarantee that for $q$ sufficiently close to the origin the orbit $\varphi(t, q)$ intersects $\Sigma_{1}$. Consequently $\pi_{0}$ is well defined in a sufficiently small neighbourhood of the origin of $\Sigma$.

We note that $a$ is a hyperbolic singular point having the straight line $z_{2}=z_{3}=0$ (in the local chart $\left.U_{1}\right)$ as the unstable manifold. Let $W^{s}(a)$ be the stable manifold of $a$. All the orbits of $X$ passing through points of $\Sigma_{1} \backslash W^{s}(a)$, that are sufficiently close to $q_{1}$ intersect either $\Sigma_{2}$ or $\Sigma_{2}^{\prime}$. Let $A_{1}$ and $A_{1}^{\prime}$ be the set of points of $\Sigma_{1} \backslash W^{s}(a)$ associated to orbits that intersect $\Sigma_{2}$ and $\Sigma_{2}^{\prime}$, respectively (see Fig. 4). We can consider two diffeomorphisms, the diffeomorphism $\pi_{1}: A_{1} \subset \Sigma_{1} \rightarrow \Sigma_{2}$ defined by $\pi_{1}(q)=p$, where $p$ is the point at which the orbit $\varphi(t, q)$ intersects the cross section $\Sigma_{2}$ for the first time; and the diffeomporphism $\pi_{1}^{\prime}: A_{1}^{\prime} \subset \Sigma_{1} \rightarrow \Sigma_{2}^{\prime}$ defined in a similar way.

We consider the diffeomorphism $\pi_{2}: \Sigma_{2} \rightarrow \bar{\Sigma}$ defined by $\pi_{2}(q)=p$, where $p$ is the point at which the orbit $\varphi(t, q)$ intersects the cross section $\bar{\Sigma}$ for the first time. Clearly if $q$ is sufficiently close to $q_{2}$, then $\pi_{2}$ is well defined. We consider also the diffeomorphism $\pi_{2}^{\prime}: \Sigma_{2}^{\prime} \rightarrow \bar{\Sigma}^{\prime}$ defined in a similar way.

For $\varepsilon>0$ sufficiently small we define $D_{\varepsilon}=$ $\left\{\left(0, y_{0}, z_{0}\right) \in \Sigma \backslash W: 0<y_{0}^{2}+z_{0}^{2}<\varepsilon^{2}\right\}$, where $W=$ $\pi_{0}^{-1}\left(W^{s}(a) \cap \Sigma_{1}\right)$. We note that $W$ is a differential curve in $\Sigma$ passing through the origin that separates the punctured disc $\left\{\left(0, y_{0}, z_{0}\right) \in \Sigma: 0<y_{0}^{2}+z_{0}^{2}<\right.$ $\left.\varepsilon^{2}\right\}$ into two components, $D_{1}^{\varepsilon}$ and $D_{2}^{\varepsilon}$. We consider the Poincaré maps $\pi: \Sigma \longrightarrow \bar{\Sigma}$ and $\pi^{\prime}: \Sigma \longrightarrow \bar{\Sigma}^{\prime}$ defined by $\pi=\pi_{2} \circ \pi_{1} \circ \pi_{0}$ and $\pi^{\prime}=\pi_{2}^{\prime} \circ \pi_{1}^{\prime} \circ \pi_{0}$. It is easy to see that if $\varepsilon>0$ is sufficiently small, then $\pi$ and $\pi^{\prime}$ are well defined in all $D_{1}^{\varepsilon} \subset \Sigma$ and $D_{2}^{\varepsilon} \subset \Sigma$, respectively.

From condition $\left(C_{1}\right)$, the vector field $X$ 
is invariant under the symmetry $(x, y, z, t)$ $\longrightarrow(-x, y, z,-t)$, this means that if $\phi(t)=$ $(x(t), y(t), z(t))$ is an orbit of $X$, then $\psi(t)=(-x(-t), y(-t), z(-t))$ is also an orbit. This symmetry can be used in order to obtain symmetric periodic orbits in the following way. Using the symmetry it is easy to see that if $x(0)=0$, then the orbits $\phi(t)$ and $\psi(t)$ must be the same. Moreover, if there exists a time $\bar{t}>0$ such that $x(\bar{t})=0$ and $x(t) \neq 0$ for all $0<t<\bar{t}$, then the orbit is periodic with period $2 \bar{t}$. In other words, if an orbit intersects the plane of symmetry $x=0$ at two different points, then it is a periodic orbit.

We note that the sets $\Sigma$ and $\bar{\Sigma}$ and $\bar{\Sigma}^{\prime}$ belong to the plane of symmetry $x=0$. Since for $\varepsilon>0$ sufficiently small $\pi\left(D_{1}^{\varepsilon}\right) \subset \bar{\Sigma}$ and $\pi^{\prime}\left(D_{2}^{\varepsilon}\right) \subset \bar{\Sigma}^{\prime}$, all the orbits of $X$ passing through points of $D_{1}^{\varepsilon}$ (respectively, $D_{2}^{\varepsilon}$ ) intersect the plane of symmetry at two different points, one in $\Sigma$ and the other one in $\bar{\Sigma}$ (respectively, $\bar{\Sigma}^{\prime}$ ). Therefore, for $\varepsilon>0$ sufficiently small the points of $D_{\varepsilon}=D_{1}^{\varepsilon} \cup D_{2}^{\varepsilon}$ correspond to initial conditions of symmetric periodic orbits of $X$ that are close to either the heteroclinic loop $\mathcal{L}$ or the heteroclinic loop $\mathcal{L}^{\prime}$. This proves statement (a) of Theorem 1.1.

The fact that for $\varepsilon>0$ sufficiently small the points of $D_{\varepsilon}$ correspond to periodic orbits of $X$ implies that $W^{u}(b)=W^{s}(a)$. Indeed, if $W^{u}(b) \neq$ $W^{s}(a)$, then $W^{u}(b) \cap \Sigma$ would be a differentiable curve in $\Sigma$ passing through the origin different from the curve $W$. Then by statement (a) the points of $W^{u}(b) \cap D_{\varepsilon}$ would correspond to periodic orbits. But the points of $W^{u}(b)$ could not correspond to periodic orbits because the orbits passing through these points tend to $b$ when $t \rightarrow-\infty$. Therefore $W^{u}(b)=W^{s}(a)$. This proves statement (b) of Theorem 1.1 .

\section{Proof of Theorem $\mathbf{1 . 2}$}

We consider an arbitrary polynomial vector field $X=(P, Q, R)$ of even degree $n$ with

$$
\begin{aligned}
P & =\sum_{0 \leqslant i+j+k \leqslant n} a_{i j k} x^{i} y^{j} z^{k}, \\
Q & =\sum_{0 \leqslant i+j+k \leqslant n} b_{i j k} x^{i} y^{j} z^{k}
\end{aligned}
$$

$$
R=\sum_{0 \leqslant i+j+k \leqslant n} c_{i j k} x^{i} y^{j} z^{k} .
$$

Assuming that the straight line $y=z=0$ is invariant by the flow of $X$ we have that $b_{i 00}=c_{i 00}=0$ for all $i \in\{0,1, \ldots, n\}$. Imposing that the system associated to $X$ is invariant under the symmetry $\left(C_{1}\right)$ we get that $a_{i j k}=0$ for all odd $i \in\{1, \ldots, n-1\}$, and $b_{i j k}=c_{i j k}=0$ for all even $i \in\{0, \ldots, n\}$. Under these conditions the flow on the straight line $y=z=0$ is given by

$$
\dot{x}=\sum_{\substack{0 \leqslant i \leqslant n \\ i \text { even }}} a_{i 00} x^{i}=f(x) .
$$

Since we want that the flow of $X$ on the straight line $y=z=0$ does not contain any singular point, we need that the equation $f(x)=0$ has no real solutions. Moreover since the flow on this straight line must go in the increasing direction of the $x$-axis we have that $f(x)>0$ for all $x \in \mathbb{R}$. In short, after imposing conditions $\left(C_{1}\right)-\left(C_{3}\right)$, we get conditions (i) and (ii) of the theorem.

Now we analyze the vector field $X$ at infinity. We start imposing the condition $\left(C_{5}\right)$. The system in the local chart $U_{2}$ associated to the vector field $X$ is given by

$$
\begin{aligned}
& \dot{z}_{1}=-\sum_{\substack{0 \leqslant i+j+k \leqslant n \\
i \text { odd } j+k \geqslant 1}} b_{i j k} z_{1}^{i+1} z_{2}^{k} z_{3}^{n-i-j-k}+ \\
& \dot{z}_{2}=-\sum_{\substack{0 \leqslant i+j+k \leqslant n \\
0 \leqslant i+j+k \leqslant n \\
i \text { even }}} a_{i j k} z_{1}^{i} z_{2}^{k} z_{3}^{n-i-j-k}, \\
& \dot{z}_{3}=-\sum_{\substack{0 \leqslant i+j+k \geqslant 1 \\
i \text { odd, } j+k \geqslant 1}} c_{i j k} z_{1}^{i} z_{2}^{k} z_{3}^{n-i-j-k}, \\
& \sum_{\substack{0 \leqslant i+j+k \leqslant n \\
i \text { odd }, j+k \geqslant 1}} b_{i j k} z_{1}^{i} z_{2}^{k+1} z_{3}^{n-i-j-k}+
\end{aligned}
$$

Imposing that the straight line $z_{2}=z_{3}=0$ is invariant by the flow, we have that $c_{i, n-i, 0}=0$ for all odd $i \in\{1, \ldots, n-1\}$; that is, we have obtained condition (iv). On the other hand, we need that the straight line $z_{2}=z_{3}=0$ in the local chart $U_{2}$ does not contain any singular point and the flow on it goes in the decreasing direction of the $z_{1}$-axis. The flow on this straight line is given by $\dot{z}_{1}=g\left(z_{1}\right)$ 
with

$$
g\left(z_{1}\right)=\sum_{\substack{0 \leqslant i \leqslant n \\ i \text { even }}} a_{i, n-i, 0} z_{1}^{i}-\sum_{\substack{0 \leqslant i \leqslant n \\ i \text { odd }}} b_{i, n-i, 0} z_{1}^{i+1} .
$$

Then, we need that $g\left(z_{1}\right)<0$ for all $z_{1} \in \mathbb{R}$. Hence, condition (vi) is obtained.

Now we impose the condition $\left(C_{4}\right)$; that is, we impose that the origin $a$ of the chart $U_{1}$ be a hyperbolic singular point with the straight line $z_{2}=z_{3}=0$ as the local unstable manifold. The system in the local chart $U_{1}$ associated to the vector field $X$ is given by

$$
\begin{aligned}
& \dot{z}_{1}=-\sum_{\substack{0 \leqslant i+j+k \leqslant n \\
i \text { even }}} a_{i j k} z_{1}^{j+1} z_{2}^{k} z_{3}^{n-i-j-k}+ \\
& \sum_{\substack{0 \leqslant i+j+k \leqslant n \\
i}} b_{i j k} z_{1}^{j} z_{2}^{k} z_{3}^{n-i-j-k}, \\
& \dot{z}_{2}=-\sum_{0 \leqslant i+j+k \leqslant n}^{i \text { odd, } j+k \geqslant 1} a_{i j k} z_{1}^{j} z_{2}^{k+1} z_{3}^{n-i-j-k}+ \\
& \sum_{0 \leqslant i+j+k \leqslant n}^{i \text { even }} c_{i j k} z_{1}^{j} z_{2}^{k} z_{3}^{n-i-j-k} \text {, } \\
& \dot{z}_{3}=-\sum_{\substack{0 \leqslant i+j+k \leqslant n \\
i \text { even }}}^{i \text { odd, } j+k \geqslant 1} a_{i j k} z_{1}^{j} z_{2}^{k} z_{3}^{n-i-j-k+1} .
\end{aligned}
$$

Since $c_{n-1,1,0}=0$, the linear part of this system at the origin is the matrix $M$ given by

$$
\left(\begin{array}{ccc}
-a_{n 00}+b_{n-1,1,0} & b_{n-1,0,1} & 0 \\
0 & -a_{n 00}+c_{n-1,0,1} & 0 \\
0 & 0 & -a_{n 00}
\end{array}\right) .
$$

The eigenvalues of $M$ are $-a_{n 00},-a_{n 00}+b_{n-1,1,0}$ and $-a_{n 00}+c_{n-1,0,1}$, and the eigenvectors associated to these eigenvalues are $(0,0,1),(1,0,0)$ and $\left(-b_{n-1,0,1} /\left(b_{n-1,1,0}-c_{n-1,0,1}\right), 1,0\right)$, respectively. We note that the third eigenvector of $M$ is defined only when $b_{n-1,1,0}-c_{n-1,0,1} \neq 0$. The necessary and sufficient conditions for the hyperbolicity of the singular point $a$ and for $W^{u}(a)=\left\{z_{2}=z_{3}=0\right\}$, are $a_{n 00}>0,-a_{n 00}+b_{n-1,1,0}>0$ and $-a_{n 00}+c_{n-1,0,1}<$ 0 . We note that if those conditions are satisfied then $b_{n-1,1,0}-c_{n-1,0,1}>0$. So we obtain conditions (iii) and $(\mathrm{v})$. This completes the proof of Theorem 1.2.

\section{The quadratic systems}

In this section we will analyze the class of quadratic systems that satisfy conditions $\left(C_{1}\right)-\left(C_{5}\right)$. From
Theorem 1.2 the most general quadratic system satisfying conditions $\left(C_{1}\right)-\left(C_{5}\right)$ is

$$
\begin{aligned}
\dot{x} & =a_{0}+a_{1} x^{2}+a_{2} y+a_{3} y^{2}+a_{4} z+a_{5} y z+a_{6} z^{2}, \\
\dot{y} & =b_{1} x y+b_{2} x z, \\
\dot{z} & =c x z,
\end{aligned}
$$

with $a_{0}>0, a_{3}<0, b_{1}>a_{1}>0$ and $c<a_{1}$.

We note that by rescaling conveniently the time and the variables we can reduce by four the number of parameters, but we shall work with all the parameters.

The next lemma proves the final statement of Proposition 1.3. The previous part of Proposition 1.3 follows immediately from Theorems 1.1 and 1.2.

Lemma 5.1. For all the values of the parameters satisfying conditions $a_{0}>0, a_{3}<0, b_{1}>a_{1}>0$ and $c<a_{1}$, system (1) has two independent first integrals, and therefore it is integrable.

Proof. We will distinguish two cases: $c \neq 0$ and $c=0$. We start analyzing the case $c=0$. Clearly when $c=0, H_{1}=z$ is a first integral of (1), and it is easy to cheek that the function $H_{2}$ given by

$$
\begin{aligned}
& \left|b_{1} y+b_{2} z\right|^{-2 a_{1}}\left[a_{0}\left(2 a_{1}{ }^{2}-3 a_{1} b_{1}+b_{1}{ }^{2}\right)+\right. \\
& 2 a_{1}^{3} x^{2}+z\left(a_{4} b_{1}^{2}-a_{2} b_{1} b_{2}+\left(a_{6} b_{1}{ }^{2}-a_{5} b_{1} b_{2}+\right.\right. \\
& \left.\left.a_{3} b_{2}^{2}\right) z\right)+a_{1}^{2}\left(-3 b_{1} x^{2}+2\left(a_{2} y+a_{3} y^{2}+z\left(a_{4}+\right.\right.\right. \\
& \left.\left.\left.a_{5} y+a_{6} z\right)\right)\right)+a_{1}\left(b_{1}^{2} x^{2}+b_{2} z\left(a_{2}+2 a_{3} y+a_{5} z\right)-\right. \\
& \left.\left.b_{1}\left(2 a_{2} y+a_{3} y^{2}+z\left(3 a_{4}+2 a_{5} y+3 a_{6} z\right)\right)\right)\right]^{b_{1}},
\end{aligned}
$$

is another first integral of system (1). Moreover, it is immediate to verify that if $b_{1} \neq 2 a_{1}$, then the first integrals $H_{1}$ and $H_{2}$ are independent. When $b_{1}=2 a_{1}$ the function $F_{2}$ given by

$$
\begin{aligned}
& \frac{1}{2 a_{1} y+b_{2} z}\left[4 a_{0} a_{1}^{2}+4 a_{1}^{3} x^{2}-4 a_{1}^{2} a_{3} y^{2}+\right. \\
& 4 a_{1}^{2} a_{4} z-2 a_{1} a_{2} b_{2} z-2 a_{1} a_{3} b_{2} y z+4 a_{1}^{2} a_{6} z^{2}- \\
& 2 a_{1} a_{5} b_{2} z^{2}+a_{3} b_{2}{ }^{2} z^{2}-2\left(2 a_{1} y+b_{2} z\right)\left(-a_{3} b_{2} z+\right. \\
& \left.\left.a_{1}\left(a_{2}+a_{5} z\right)\right) \ln \left|2 a_{1} y+b_{2} z\right|\right],
\end{aligned}
$$

is another first integral of (1) independent with $H_{1}$ when $c=0$. 
Now we analyze the case $c \neq 0$. We can verify that when $c \neq 0$

$$
H_{1}=|z|^{-b_{1}}\left(y+\frac{b_{2} z}{b_{1}-c}\right)^{c},
$$

and the function $\mathrm{H}_{2}$ given by

$$
\begin{aligned}
& |z|^{-2 a_{1}}\left[a_{1}\left(b_{1}-2 a_{1}\right)\left(b_{1}-a_{1}\right)\left(c-2 a_{1}\right)\left(c-a_{1}\right)\right. \\
& \left(b_{1}+c-2 a_{1}\right) x^{2}-8 a_{0} a_{1}{ }^{5}+16 a_{0} a_{1}{ }^{4} b_{1}- \\
& 10 a_{0} a_{1}^{3} b_{1}^{2}+2 a_{0} a_{1}^{2} b_{1}^{3}+16 a_{0} a_{1}{ }^{4} c-30 a_{0} a_{1}^{3} b_{1} c+ \\
& 17 a_{0} a_{1}{ }^{2} b_{1}{ }^{2} c-3 a_{0} a_{1} b_{1}{ }^{3} c-10 a_{0} a_{1}{ }^{3} c^{2}+ \\
& 17 a_{0} a_{1}{ }^{2} b_{1} c^{2}-8 a_{0} a_{1} b_{1}{ }^{2} c^{2}+a_{0} b_{1}{ }^{3} c^{2}+2 a_{0} a_{1}{ }^{2} c^{3}- \\
& 3 a_{0} a_{1} b_{1} c^{3}+a_{0} b_{1}{ }^{2} c^{3}-8 a_{1}^{5} a_{2} y+12 a_{1}^{4} a_{2} b_{1} y- \\
& 4 a_{1}^{3} a_{2} b_{1}^{2} y+16 a_{1}^{4} a_{2} c y-22 a_{1}^{3} a_{2} b_{1} c y+ \\
& 6 a_{1}^{2} a_{2} b_{1}^{2} c y-10 a_{1}^{3} a_{2} c^{2} y+12 a_{1}^{2} a_{2} b_{1} c^{2} y- \\
& 2 a_{1} a_{2} b_{1}{ }^{2} c^{2} y+2 a_{1}^{2} a_{2} c^{3} y-2 a_{1} a_{2} b_{1} c^{3} y- \\
& 8 a_{1}^{5} a_{3} y^{2}+8 a_{1}^{4} a_{3} b_{1} y^{2}-2 a_{1}^{3} a_{3} b_{1}^{2} y^{2}+ \\
& 16 a_{1}{ }^{4} a_{3} c y^{2}-14 a_{1}{ }^{3} a_{3} b_{1} c y^{2}+3 a_{1}{ }^{2} a_{3} b_{1}{ }^{2} c y^{2}- \\
& 10 a_{1}^{3} a_{3} c^{2} y^{2}+7 a_{1}{ }^{2} a_{3} b_{1} c^{2} y^{2}-a_{1} a_{3} b_{1}{ }^{2} c^{2} y^{2}+ \\
& 2 a_{1}^{2} a_{3} c^{3} y^{2}-a_{1} a_{3} b_{1} c^{3} y^{2}-8 a_{1}^{5} a_{4} z+ \\
& 16 a_{1}{ }^{4} a_{4} b_{1} z-10 a_{1}{ }^{3} a_{4} b_{1}{ }^{2} z+2 a_{1}{ }^{2} a_{4} b_{1}{ }^{3} z- \\
& 4 a_{1}{ }^{4} a_{2} b_{2} z+6 a_{1}^{3} a_{2} b_{1} b_{2} z-2 a_{1}^{2} a_{2} b_{1}^{2} b_{2} z+ \\
& 12 a_{1}^{4} a_{4} c z-22 a_{1}^{3} a_{4} b_{1} c z+12 a_{1}^{2} a_{4} b_{1}^{2} c z- \\
& 2 a_{1} a_{4} b_{1}{ }^{3} c z+6 a_{1}{ }^{3} a_{2} b_{2} c z-8 a_{1}^{2} a_{2} b_{1} b_{2} c z+ \\
& 2 a_{1} a_{2} b_{1}^{2} b_{2} c z-4 a_{1}^{3} a_{4} c^{2} z+6 a_{1}^{2} a_{4} b_{1} c^{2} z- \\
& 2 a_{1} a_{4} b_{1}{ }^{2} c^{2} z-2 a_{1}{ }^{2} a_{2} b_{2} c^{2} z+2 a_{1} a_{2} b_{1} b_{2} c^{2} z- \\
& 8 a_{1}{ }^{5} a_{5} y z+12 a_{1}{ }^{4} a_{5} b_{1} y z-4 a_{1}{ }^{3} a_{5} b_{1}{ }^{2} y z- \\
& 8 a_{1}^{4} a_{3} b_{2} y z+4 a_{1}^{3} a_{3} b_{1} b_{2} y z+12 a_{1}{ }^{4} a_{5} c y z- \\
& 18 a_{1}^{3} a_{5} b_{1} c y z+6 a_{1}{ }^{2} a_{5} b_{1}{ }^{2} c y z+12 a_{1}{ }^{3} a_{3} b_{2} c y z- \\
& 6 a_{1}^{2} a_{3} b_{1} b_{2} c y z-4 a_{1}^{3} a_{5} c^{2} y z+6 a_{1}^{2} a_{5} b_{1} c^{2} y z- \\
& 2 a_{1} a_{5} b_{1}{ }^{2} c^{2} y z-4 a_{1}^{2} a_{3} b_{2} c^{2} y z+2 a_{1} a_{3} b_{1} b_{2} c^{2} y z- \\
& 8 a_{1}{ }^{5} a_{6} z^{2}+16 a_{1}{ }^{4} a_{6} b_{1} z^{2}-10 a_{1}{ }^{3} a_{6} b_{1}{ }^{2} z^{2}+ \\
& 2 a_{1}{ }^{2} a_{6} b_{1}{ }^{3} z^{2}-4 a_{1}{ }^{4} a_{5} b_{2} z^{2}+6 a_{1}{ }^{3} a_{5} b_{1} b_{2} z^{2}- \\
& 2 a_{1}^{2} a_{5} b_{1}^{2} b_{2} z^{2}-4 a_{1}^{3} a_{3} b_{2}^{2} z^{2}+2 a_{1}^{2} a_{3} b_{1} b_{2}^{2} z^{2}+ \\
& 8 a_{1}{ }^{4} a_{6} c z^{2}-14 a_{1}{ }^{3} a_{6} b_{1} c z^{2}+7 a_{1}{ }^{2} a_{6} b_{1}{ }^{2} c z^{2}- \\
& a_{1} a_{6} b_{1}{ }^{3} c z^{2}+2 a_{1}{ }^{3} a_{5} b_{2} c z^{2}-3 a_{1}{ }^{2} a_{5} b_{1} b_{2} c z^{2}+ \\
& a_{1} a_{5} b_{1}^{2} b_{2} c z^{2}+2 a_{1}^{2} a_{3} b_{2}^{2} c z^{2}-a_{1} a_{3} b_{1} b_{2}^{2} c z^{2}- \\
& \left.2 a_{1}{ }^{3} a_{6} c^{2} z^{2}+3 a_{1}{ }^{2} a_{6} b_{1} c^{2} z^{2}-a_{1} a_{6} b_{1}{ }^{2} c^{2} z^{2}\right]^{c},
\end{aligned}
$$

are two first integrals of (1). Moreover if $b_{1} \neq 2 a_{1}$ and $c \neq 2 a_{1}-b_{1}$, then these two first integrals are independent. When $b_{1}=2 a_{1}$ the function $F_{2}$ given by

$$
\begin{aligned}
& |z|^{-2 a_{1}}\left[a_{1}\left(a_{1}-c\right) c\left(c-2 a_{1}\right)^{2} x^{2}-a_{3}\left(a_{1}-c\right) c\right. \\
& \left(2 a_{1} y-c y+b_{2} z\right)^{2}-2 a_{1}\left(a_{1}-c\right)\left(2 a_{1} a_{5}-\right. \\
& \left.2 a_{3} b_{2}-a_{5} c\right) z\left(2 a_{1} y-c y+b_{2} z\right)+ \\
& c\left(a_{0}\left(a_{1}-c\right)\left(c-2 a_{1}\right)^{2}+a_{1} z\left(2 a_{2} b_{2} c+2 a_{4} c^{2}+\right.\right. \\
& a_{3} b_{2}^{2} z+a_{5} b_{2} c z+a_{6} c^{2} z+4 a_{1}^{2}\left(a_{4}+a_{6} z\right)- \\
& \left.\left.2 a_{1}\left(a_{2} b_{2}+3 a_{4} c+a_{5} b_{2} z+2 a_{6} c z\right)\right)\right)- \\
& \left.2 a_{1} a_{2}\left(a_{1}-c\right)\left(2 a_{1}-c\right)\left(2 a_{1} y-c y+b_{2} z\right) \ln |z|\right]^{c}
\end{aligned}
$$

is another first integral of (1) independent with $H_{1}$; and when $c=2 a_{1}-b_{1}$ the function $G_{2}$ given by

$$
\begin{aligned}
& |z|^{-2 a_{1}}\left[2 a_{0}\left(a_{1}-b_{1}\right)^{2}\left(2 a_{1}-b_{1}\right) b_{1}+2 a_{1}\right. \\
& \left(a_{1}-b_{1}\right)^{2}\left(2 a_{1}-b_{1}\right) b_{1} x^{2}+a_{1}\left(8 a_{1}^{3} a_{4} z-\right. \\
& 2 a_{1} b_{1}\left(4 a_{2}\left(b_{1} y+b_{2} z\right)+a_{3} y\left(3 b_{1} y+2 b_{2} z\right)+\right. \\
& \left.z\left(-8 a_{4} b_{1}-3 a_{6} b_{1} z+a_{5} b_{2} z\right)\right)+b_{1}^{2}\left(4 a _ { 2 } \left(b_{1} y\right.\right. \\
& \left.+b_{2} z\right)+2 a_{3} y\left(b_{1} y+b_{2} z\right)+z\left(-4 a_{4} b_{1}-\right. \\
& \left.\left.2 a_{6} b_{1} z+a_{5} b_{2} z\right)\right)+4 a_{1}^{2}\left(a_{2}\left(b_{1} y+b_{2} z\right)+\right. \\
& \left.\left.b_{1}\left(a_{3} y^{2}-z\left(5 a_{4}+a_{6} z\right)\right)\right)\right)-2 a_{1} b_{1}\left(a_{1} a_{5}-\right. \\
& \left.\left.a_{5} b_{1}+a_{3} b_{2}\right) z\left(2 a_{1} y-2 b_{1} y-b_{2} z\right) \ln |z|\right]^{2 a_{1}-b_{1}}
\end{aligned}
$$

is also a first integral of (1) independent with $H_{1}$. We note that the case $b_{1}=2 a_{1}$ and $c=2 a_{1}-b_{1}$ is not possible because $c \neq 0$.

Next we give a complete description of the global phase portrait of a particular quadratic system satisfying conditions $\left(C_{1}\right)-\left(C_{5}\right)$. For our analysis we choose the system

$$
\dot{x}=1+\frac{x^{2}}{2}-y^{2}, \quad \dot{y}=x y, \quad \dot{z}=-x z .
$$

The phase portrait of system (2) is the description of $\mathbb{R}^{3}$ (the domain of definition of system (2)) as union of its orbits. It is well known that all the orbits of a system of differential equations are either an equilibrium point $\{p\}$, a periodic orbit diffeomorphic to $\mathbb{S}^{1}$, or a curve diffeomorphic to $\mathbb{R}$.

By the proof of Lemma 5.1, system (2) is integrable with the two independent first integrals

$$
H_{1}=y z, \quad \text { and } \quad H_{2}=\left(2+x^{2}+2 y^{2}\right) z .
$$




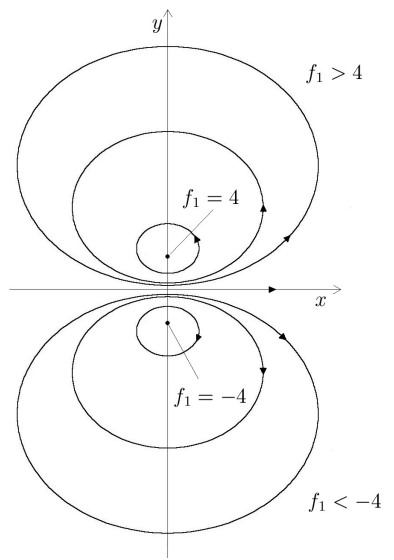

Fig. 5. Phase portrait of system (2) on the invariant plane $z=0$.

Clearly the sets $I_{h_{1}}=\left\{(x, y, z) \in \mathbb{R}^{3}: H_{1}=h_{1}\right\}$, $I_{h_{2}}=\left\{(x, y, z) \in \mathbb{R}^{3}: H_{2}=h_{2}\right\}$ and $I_{h_{1} h_{2}}=$ $\left\{(x, y, z) \in \mathbb{R}^{3}: H_{1}=h_{1}, H_{2}=h_{2}\right\}$ are invariant by the flow of (2). Moreover the phase portrait of (2) is essentially given by the foliation of the phase space of (2) by the sets $I_{h_{1} h_{2}}$ depending on the values of $h_{1}$ and $h_{2}$. We note that the first integrals $H_{1}$ and $H_{2}$ are independent for all $(x, y, z) \in \mathbb{R}^{3}$ except for the points of the plane $z=0$ and the points of the straight lines $x=0, y= \pm 1$. It is easy to see that the straight lines $x=0, y= \pm 1$ are lines of equilibrium points of system (2). On the other hand, the plane $z=0$ is invariant by the flow of (2).

We start analyzing the flow on $z=0$. System (2) restricted to this plane is

$$
\dot{x}=1+\frac{x^{2}}{2}-y^{2}, \quad \dot{y}=x y .
$$

This system has the first integral $F_{1}=\left(2+x^{2}+\right.$ $\left.2 y^{2}\right) / y$. Computing the sets $I_{f_{1}}=\left\{(x, y) \in \mathbb{R}^{2}\right.$ : $\left.F_{1}=f_{1}\right\}$ we see that $I_{f_{1}}$ is diffeomorphic to

$$
\begin{array}{ll}
\emptyset & \text { if }\left|f_{1}\right|<4, \\
\{p\} & \text { if }\left|f_{1}\right|=4, \\
\mathbb{S}^{1} & \text { if }\left|f_{1}\right|>4,
\end{array}
$$

where $p=(0,-1)$ if $f_{1}=-4$ and $p=(0,1)$ if $f_{1}=$ 4. The phase portrait of system (3) is described in Fig. 5.

Now we analyze the foliation of the space $E=$ $\left\{(x, y, z) \in \mathbb{R}^{3}: z \neq 0\right\}$ by the sets $I_{h_{1}}$ and $I_{h_{1} h_{2}}$. First we compute the sets $I_{h_{1}}$. Clearly, $I_{h_{1}}=\left\{(x, y, z) \in \mathbb{R}^{3}: y=h_{1} / z\right\}$. We note that this

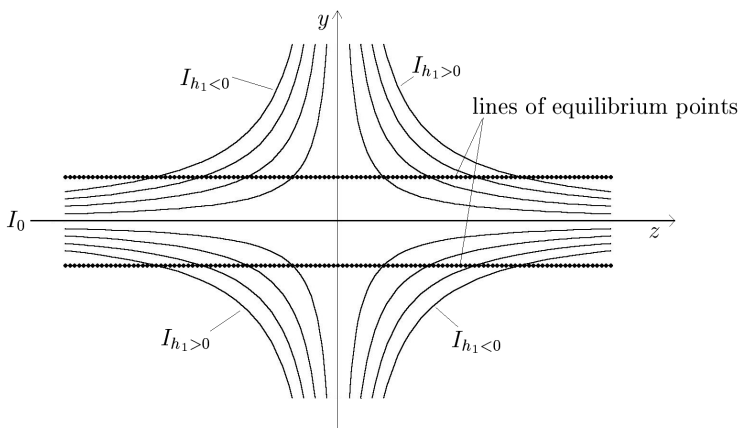

Fig. 6. Projection of the sets $I_{h_{1}}$ on the plane $x=0$.

set is homeomorphic to two copies of $\mathbb{R}^{2}$ if $h_{1} \neq 0$, and it is the plane $y=0$ when $h_{1}=0$, see Fig. 6 .

Fixed a value of $h_{1}$, we analyze the set $I_{h_{1} h_{2}}$ which is given by

$$
\left\{(x, y, z) \in \mathbb{R}^{3}: x= \pm \sqrt{\frac{h_{2} z-2 h_{1}^{2}-2 z^{2}}{z^{2}}}\right\} .
$$

Let $f\left(h_{1}, h_{2}, z\right)=h_{2} z-2 h_{1}^{2}-2 z^{2}$ and $g\left(h_{1}, h_{2}, z\right)=$ $\left(h_{2} z-2 h_{1}^{2}-2 z^{2}\right) / z^{2}$. It is easy to check that if $h_{2}^{2}-16 h_{1}^{2}<0$ then $f\left(h_{1}, h_{2}, z\right)<0$ for all $z \in \mathbb{R}$. If $h_{2}^{2}-16 h_{1}^{2}=0$, then $f\left(h_{1}, h_{2}, z\right)<0$ for all $z \neq h_{2} / 4$ and $f\left(h_{1}, h_{2}, h_{2} / 4\right)=0$. Finally if $h_{2}^{2}-16 h_{1}^{2}>$ 0 , then $f\left(h_{1}, h_{2}, z\right)>0$ for all $z \in(\alpha, \beta)=$ $\left(\left(h_{2}-\sqrt{{h_{2}}^{2}-16 h_{1}^{2}}\right) / 4,\left(h_{2}+\sqrt{{h_{2}}^{2}-16 h_{1}{ }^{2}}\right) / 4\right)$ and $f\left(h_{1}, h_{2}, \alpha\right)=f\left(h_{1}, h_{2}, \beta\right)=0$. We note that if $h_{1}=0$, then $\alpha=0$ and the function $g\left(h_{1}, h_{2}, z\right)$ is not defined for $z=0$. In particular, if $h_{1}=h_{2}=0$, then $g(0,0, z)<0$ for all $z \in \mathbb{R}$. If $h_{1}=0$ and $h_{2}>0$ (respectively, $\left.h_{2}<0\right)$, then $g\left(0, h_{2}, z\right)>0$ for all $z \in\left(0, h_{2} / 2\right)$, $g\left(0, h_{2}, h_{2} / 2\right)=0$ and $\lim _{z \rightarrow 0^{+}} g\left(0, h_{2}, z\right)=+\infty$ (respectively, $g\left(0, h_{2}, z\right)>0$ for all $z \in\left(h_{2} / 2,0\right)$, $g\left(0, h_{2}, h_{2} / 2\right)=0$ and $\left.\lim _{z \rightarrow 0^{-}} g\left(0, h_{2}, z\right)=+\infty\right)$. In short, $I_{h_{1} h_{2}}$ is diffeomorphic to

$\emptyset \quad$ if either $h_{2}^{2}-16 h_{1}^{2}<0$ or $h_{1}=h_{2}=0$,

$\{p\} \quad$ if $h_{2}^{2}-16 h_{1}^{2}=0$ and $h_{1} \neq 0$,

$\mathbb{S}^{1} \quad$ if $h_{2}^{2}-16 h_{1}^{2}>0$ and $h_{1} \neq 0$,

$\mathbb{R} \quad$ if $h_{2}^{2}-16 h_{1}^{2}>0$ and $h_{1}=0$,

where $p=\left(0,4 h_{1} / h_{2}, h_{2} / 4\right)$. This result is summarized in Fig. 7.

We note that the plane $y=0$ is invariant by the flow of (2) and it corresponds to the invariant set $I_{h_{1}}$ with $h_{1}=0$. In Fig. 8(a) we give the foliation of $I_{0}$ by the invariant sets $I_{0 h_{2}}$. In Fig. 8(b) we give the foliation of the set $I_{h_{1}}$, for a fixed value 


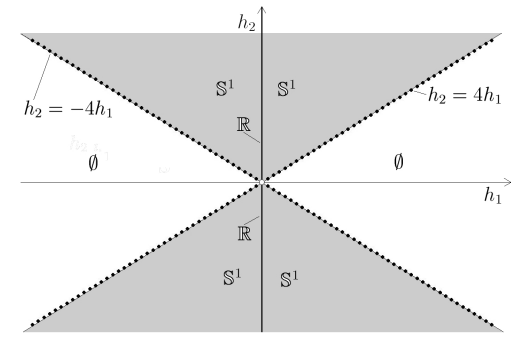

Fig. 7. Topology of the sets $I_{h_{1} h_{2}}$.

of $h_{1} \neq 0$, by the invariant sets $I_{h_{1} h_{2}}$. We remark that in Fig. 8(b) we only plot the coordinates $x$ and $z$, the coordinate $y$ can be obtained through the equation $H_{1}=h_{1}$. Finally in Fig. 9 we give a 3 -dimensional representation of the phase portrait of system (2) restricted to $E_{1}=I_{h_{1}} \cap\{(x, y, z) \in$ $\left.\mathbb{R}^{3}: y \geqslant 0, z \geqslant 0\right\}$ for $h_{1}=0$, and for $h_{1} \neq 0$. Since system (2) is invariant under symmetries

$$
\begin{aligned}
& (x, y, z, t) \longrightarrow(-x, y, z,-t), \\
& (x, y, z, t) \longrightarrow(-x,-y,-z, t), \\
& (x, y, z, t) \longrightarrow(-x, y,-z,-t),
\end{aligned}
$$

the phase portrait on the sets $I_{h_{1}} \cap\left\{(x, y, z) \in \mathbb{R}^{3}\right.$ : $y \geqslant 0, z \leqslant 0\}, I_{h_{1}} \cap\left\{(x, y, z) \in \mathbb{R}^{3}: y \leqslant 0, z \geqslant\right.$ $0\}$ and $I_{h_{1}} \cap\left\{(x, y, z) \in \mathbb{R}^{3}: y \leqslant 0, z \leqslant 0\right\}$ is topologically the same.

From the analysis of the phase portrait of system (2), we see that the set of non-periodic orbits of the system has zero Lebesgue measure.

\section{Acknowledgement.}

The authors are partially supported by the grants MCYT number BFM2002-04236-C02-02 and CIRIT number 2001SGR00173.

\section{References}

Buzzi, C.A., Llibre, J. and \& Medrano,J.C. [2004] "Lymit cycles for a class of reversible quadratic vector field on $\mathbb{R}^{3}$," Preprint.

Cima, A. \& Llibre, J. [1990] "Bounded polynomial vector fields," Trans. Amer. Math. Soc. 318, $557-579$.

Llibre, J., MacKay, R.S. \& Rodríguez, G. [2004] "Periodic dynamics bifurcating from infinity," Preprint.

Newell, A.C., Rand, D.A. \& Russell, D. [1988] "Tur-

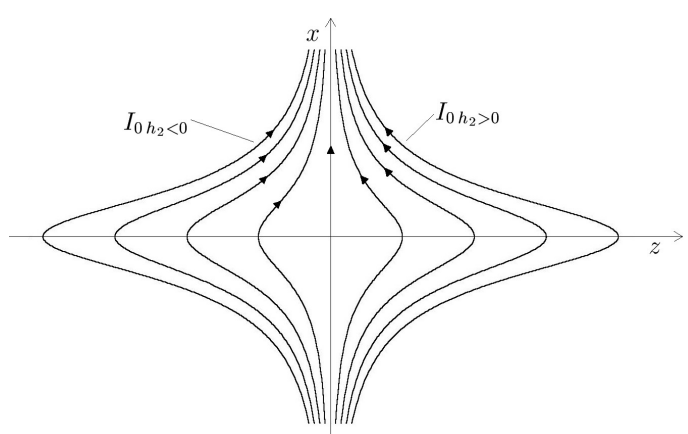

(a) $h_{1}=0$

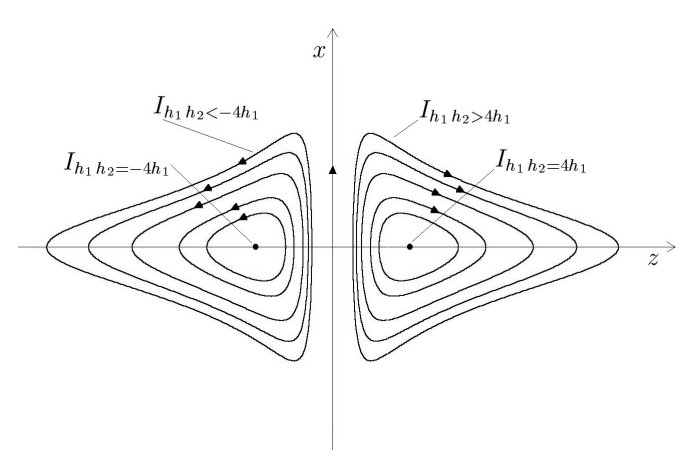

(b) $h_{1} \neq 0$

Fig. 8. Foliation of the sets $I_{h_{1}}$ by the invariant sets $I_{h_{1} h_{2}}$.

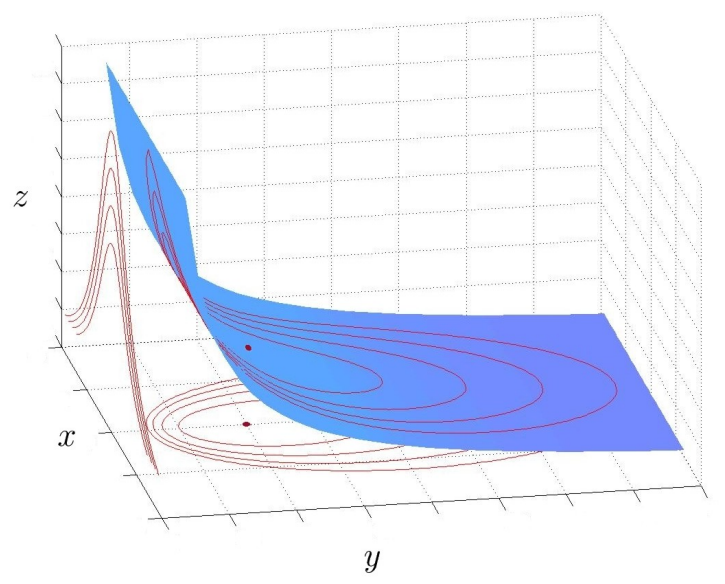

Fig. 9. The phase portrait of (2) on the set $E_{1}$. 
bulent transport and the random ocurrence of coherent events," Phys. D 33, 281-303. 